\title{
Bioavailability, tissue distribution, and excretion characteristics of the novel carbonic anhydrase inhibitor tolsultazolamide in rats
}

\author{
Jin-da WANG ${ }^{1,2,3}$, Yong-ping $\mathrm{SHI}^{2,3}$, Jing $\mathrm{YIN}^{2}$, Zhi-yuan $\mathrm{PAN}^{1}$, Wen-yu CUI ${ }^{1}$, Yan-fang ZHANG ${ }^{1}$, Hai WANG ${ }^{1, *}$ \\ ${ }^{1}$ Institute of Health and Environmental Medicine, Academy of Military Medical Science, Beijing 100850, China; ${ }^{2}$ Thadweik Academy of \\ Medicine, Beijing 100039, China; ${ }^{3}$ NhwaThad Pharmaceutical Co, Ltd, Xuzhou 221007, China
}

Aim: Tolsultazolamide, a novel carbonic anhydrase inhibitor, is designed for the prophylaxis and treatment of acute mountain sickness. The aim of this study was to investigate the pharmacokinetics, tissue distribution, and excretion characteristics of tolsultazolamide and the sex difference in pharmacokinetics in rats.

Methods: For pharmacokinetic study, rats were intravenously injected tolsultazolamide at 1 and $2 \mathrm{mg} / \mathrm{kg}$ or orally administered tolsultazolamide at 20,40 , or $80 \mathrm{mg} / \mathrm{kg}$ ) in a pharmacokinetic study. The concentrations of tolsultazolamide in plasma were determined with high-performance liquid chromatography, with a liquid-liquid extraction. For tissue distribution study, tolsultazolamide (80 mg/kg) was orally administered to overnight fasted rats (six per group and three per sex). Samples were collected from the brain, heart, lung, liver, spleen, muscle, kidney, stomach, fat, intestines, pancreas and sexual gland. For excretion study, tolsultazolamide (40 mg/kg) was orally administered to 6 rats (three per sex). The urine, feces, and bile samples were collected at 24, 48, and72 h.

Results: After its intravenous administration, tolsultazolamide was rapidly eliminated from the plasma, with $T_{1 / 2}$ of about $60-90$ min. The $\mathrm{AUC}_{0-\mathrm{t}}$ and the initial concentration (CO) values were proportional to the intravenous doses. After its oral administration, tolsultazolamide showed dose-independent pharmacokinetic characteristics, with $T_{\max }$ and $T_{1 / 2}$ of approximately $2 \mathrm{~h}$ and $5-7 \mathrm{~h}$, respectively, and good oral absolute bioavailability of about $60 \%$. Tolsultazolamide was distributed widely in various tissues. The highest tolsultazolamide levels were detected in the stomach, intestine, spleen, lung, and kidney. Total excretion of unchanged tolsultazolamide in the urine, feces, and bile was less than $2 \%$. The $C_{\max }$ and AUC of tolsultazolamide were significantly higher in female rats than those in male rats. Clearance and volume of distribution were greater in male rats than those in female rats. The oral absolute bioavailability was also significantly different between female rats (about 83\%) and male rats (about 37\%).

Conclusion: Tolsultazolamide was well absorbed and widely distributed in the rat, and very little of the unchanged form was excreted. Sex had a significant effect on the pharmacokinetics of tolsultazolamide.

Keywords: carbonic anhydrase inhibitors; tolsultazolamide; pharmacokinetics; tissue distribution; excretion; sex difference

Acta Pharmacologica Sinica (2014) 35: 275-282; doi: 10.1038/aps.2013.146; published online 16 Dec 2013

\section{Introduction}

Acute mountain sickness (AMS) is a common disease occurring at high altitudes. It is caused by hypoxia and characterized by headache, weakness, fatigue, nausea, insomnia, and depressed appetite. Although AMS does not seem serious, if untreated it can progress to the life-threatening conditions of high-altitude pulmonary edema and high-altitude cerebral edema $^{[1-4]}$. Because acute exposure to high altitudes is increasingly common for both work- and travel-related reasons, AMS is an issue of national and international concern.

\footnotetext{
* To whom correspondence should be addressed.

E-mail wh9588@sina.com

Received 2013-05-18 Accepted 2013-09-09
}

The carbonic anhydrase inhibitor acetazolamide is now widely accepted as an effective drug for the prophylaxis and treatment of AMS, but the high incidence of its adverse effects, including paresthesia, diuresis, and dysgeusia, limits its application $^{[5-8]}$. Therefore, an effective and safe drug for the prevention and treatment of AMS is urgently required. Tolsultazolamide (Figure 1A), a new derivative of acetazolamide (Figure 1B), is a novel carbonic anhydrase inhibitor discovered by our research team. The results of animal experiments have indicated that it improves the endurance of mice under hypoxic conditions and may function more effectively than acetazolamide $^{[9,10]}$.

Pharmacokinetic studies play a very important role in drug discovery and development, not only to support toxicological 
A

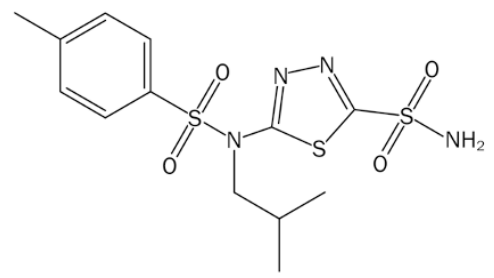

B

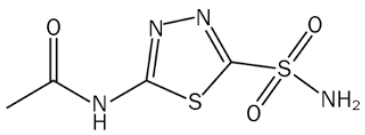

Figure 1. Structures of tolsultazolamide (A) and acetazolamide (B).

and clinical studies but also to optimize drug candidates ${ }^{[1]}$. Therefore, the objective of the current study was to examine the pharmacokinetics, tissue distribution, and excretion characteristics of tolsultazolamide in rats. The influence of sex on the pharmacokinetics of tolsultazolamide was also studied.

\section{Materials and methods Reagents}

Tolsultazolamide (purity $>98.0 \%$ ) was prepared by Academy of Military Medical Sciences (Beijing, China). Phenacetin (internal standard, IS, purity $>98.0 \%$ ) was purchased from Sigma Chemical Co (St Louis, CA, USA). Methanol of HPLC grade was obtained from Anhui Fulltime Specialized Solvents \& Reagents Co Ltd (Anhui, China). Ultra-pure water was prepared using a Milli-Q water purification system (Millipore, USA). Other reagents and solvents were of analytical grade.

\section{Instrumentation and analytical conditions}

The RP-HPLC analysis was performed on a Dionex HPLC system equipped with an ultraviolet detector (Dionex Corp, USA). Chromatographic separation was achieved using a Zorbax Eclipse Plus $C_{18}$ column $(4.6 \mathrm{~mm} \times 250 \mathrm{~mm}, 5 \mu \mathrm{m})$ coupled with a pre-column Zorbax Eclipse Plus $\mathrm{C}_{18}(4.6 \mathrm{~mm} \times 12.5 \mathrm{~mm}$, $5 \mu \mathrm{m})$ at temperature of $25^{\circ} \mathrm{C}$. The mobile phase consisted of water (contained $0.1 \%$ acetic acid) and methanol $(42: 58, v / v)$, at a flow rate of $1.0 \mathrm{~mL} / \mathrm{min}$. The sample injection volume was $20 \mu \mathrm{L}$, and the detection wavelength was $285 \mathrm{~nm}$.

\section{Animals}

Adult wistar rats [200 \pm 20 g, certificate №: SCXK-(Military) 2007-004] of both sexes were obtained from the Experimental Animal Center of the Academy of Military Medical Sciences (AMMS, Beijing, China). All animal experiments were performed in accordance with the Animal Care and Use Guidelines set by AMMS Animal Care and Use Committee.

\section{Pharmacokinetic study \\ Oral administration}

Three groups of rats (six per group and three per sex) were orally administered tolsultazolamide (suspended in $0.5 \%$ CMC-Na) at a dose of 20, 40, or $80 \mathrm{mg} / \mathrm{kg}$. Blood samples (about $0.4 \mathrm{~mL}$ at each time point) were collected through the ophthalmic venous plexus at $20 \mathrm{~min}$, and 1, 1.5, 2, 2.5, 3, 3.5, 4, $6,8,12,24$, and $32 \mathrm{~h}$ after the oral administration of the drug.
All blood samples were immediately centrifuged at $1500 \times \mathrm{g}$ for $10 \mathrm{~min}$ and the plasmas were frozen at $-20^{\circ} \mathrm{C}$ until analysis.

\section{Intravenous administration}

Two groups of rats (six per group and three per sex) were intravenously injected with tolsultazolamide (dissolved with $1 \%$ DMSO and $1 \%$ cremophor) at a dose of 1 or $2 \mathrm{mg} / \mathrm{kg}$. Blood samples (about $0.4 \mathrm{~mL}$ at each time point) were collected through the ophthalmic venous plexus at 2, 5, 10, 15, 30, 45,60 , and $120 \mathrm{~min}$ after the intravenous injection of the drug. All blood samples were immediately centrifuged at $1500 \times \mathrm{g}$ for $10 \mathrm{~min}$ and the plasmas were frozen at $-20^{\circ} \mathrm{C}$ until analysis.

\section{Tissue distribution study}

Tolsultazolamide $(80 \mathrm{mg} / \mathrm{kg})$ was orally administered to four groups of overnight fasted rats (six per group and three per sex). Samples were collected at $0.5,2,8$, and $24 \mathrm{~h}$ post-dosing from the brain, heart, lung, liver, spleen, muscle, kidney, stomach, fat, intestines, pancreas and sexual gland, thoroughly rinsed with physiological saline, and then blotted dry with filter paper and stored at $-20^{\circ} \mathrm{C}$.

\section{Excretion study}

\section{Urine and feces excretion}

After oral administration of tolsultazolamide $(40 \mathrm{mg} / \mathrm{kg})$, six rats (three per sex) were immediately placed in metabolism cages, and samples of urine and feces were collected separately and quantitatively at the following intervals: $0-24 \mathrm{~h}$, 24-48 $\mathrm{h}$ and $48-72 \mathrm{~h}$. The volumes of the urine samples were recorded. Fecal samples were dried and weighed, and then homogenized with 10 -fold volumes of water for assay.

\section{Bile excretion}

Six rats (three per sex) were anesthetized with $10 \%$ chloral hydrate $(0.3 \mathrm{~mL} / 100 \mathrm{~g})$ and bile duct cannulation was performed to allow bile collection. Bile samples were collected at intervals of $0-6 \mathrm{~h}, 6-12 \mathrm{~h}, 12-18 \mathrm{~h}$, and $18-24 \mathrm{~h}$ after the oral administration of tolsultazolamide $(40 \mathrm{mg} / \mathrm{kg})$. The volumes of the bile samples were measured for analysis.

\section{Sample pretreatment}

For pharmacokinetic study, $150 \mu \mathrm{L}$ plasma mixed with $20 \mu \mathrm{L}$ IS $(10 \mu \mathrm{g} / \mathrm{mL}$ phenacetin methanol solutions) were vortexed for $30 \mathrm{~s}$ and then extracted by $1 \mathrm{~mL}$ dichloromethane for 3 min. The substratum was transferred to a clean tube after centrifuged at $1500 \times g$ for $5 \mathrm{~min}$, and then dried by $\mathrm{N}_{2}$ at $40^{\circ} \mathrm{C}$. The residue was reconstituted with $50 \mu \mathrm{L}$ mobile phase and centrifuged at $9000 \times g$ for $5 \mathrm{~min}$, and $20 \mu \mathrm{L}$ of the supernatant was injected into the HPLC system for analysis.

For tissue distribution study, small slices of tissues were individually homogenized with 4-fold volumes of physiological saline. A $300 \mu \mathrm{L}$ homogenate spiked with $20 \mu \mathrm{L}$ IS and 100 $\mu \mathrm{L} 0.1 \mathrm{~mol} / \mathrm{L} \mathrm{HCl}$ were vortexed for $30 \mathrm{~s}$ and then extracted by $1 \mathrm{~mL}$ dichloromethane for $3 \mathrm{~min}$. Other operations were the same as pharmacokinetic study.

For excretion study, $150 \mu \mathrm{L}$ (urine or bile) or $100 \mu \mathrm{L}$ feces 
homogenate of each interval sample was extracted by $1 \mathrm{~mL}$ dichloromethane for $3 \mathrm{~min}$. Other operations were the same as pharmacokinetic study.

\section{Pharmacokinetic and statistical analysis}

Pharmacokinetic parameters of tolsultazolamide were calculated using non-compartmental methods (WinNolin ver 5.2, Pharsight Corp, Mountain View, CA, USA). The maximum plasma concentrations $\left(C_{\max }\right)$ and the time to reach the maximum concentrations $\left(T_{\max }\right)$ were obtained directly from the observed data. The elimination rate constant $\left(k_{\mathrm{e}}\right)$ was determined by linear regression of the terminal portion of plasma concentration-time data. The elimination half-life $\left(T_{1 / 2}\right)$ was calculated as $0.693 / k_{\mathrm{e}}$. The area under plasma concentrationtime curve $\left(\mathrm{AUC}_{0-\mathrm{t}}\right)$ to the last measurable plasma concentration $\left(C_{t}\right)$ was estimated by the linear trapezoidal rule. The area under the plasma concentration-time curve to time infinity $\left(\mathrm{AUC}_{0-\infty}\right)$ was calculated as $\mathrm{AUC}_{0-\infty}=\mathrm{AUC}_{0-\mathrm{t}}+\mathrm{C}_{\mathrm{t}} / k_{\mathrm{e}}$. The mean residence time (MRT) was calculated as $\mathrm{AUMC}_{0-\infty} / \mathrm{AUC}_{0-\infty}$. The total body clearance (CL) was calculated as Dose/ $\mathrm{AUC}_{0-\infty}$. The apparent volume of distribution $\left(V_{\mathrm{d}}\right)$ was calculated as $\mathrm{CL} / k_{\mathrm{e}}^{[12,13]}$.

Dose-proportionality was evaluated by comparison of the dose-normalized $\mathrm{AUC}_{0-\mathrm{t}}$ and $\mathrm{C}_{\max }$ across different dosage levels and linear regression analysis ${ }^{[12,13]}$. Data were expressed as mean \pm standard deviation (SD). The significance of difference was assessed by Student's $t$-test between two groups and oneway variance analysis (ANOVA) between three groups. A $P$-value $<0.05$ was considered statistically significant.

\section{Results}

\section{Method validation}

Under the chromatographic conditions, tolsultazolamide and IS were resolved well and were free from endogenous interference. The retention times of tolsultazolamide and IS were approximately $15 \mathrm{~min}$ and $5.5 \mathrm{~min}$, respectively (Figure 2). The standard curves showed excellent linearity, with correlation coefficients greater than 0.99 . The lower limit was defined as the lowest concentration at which both the precision and accuracy were less than or equal to $20 \%$ (Table S1 and S2). The inter- and intra-day precision (RSD) were below 15\%, and the accuracy was in the range of $85 \%-115 \%$. The extraction recoveries of both tolsultazolamide and IS were greater than $70 \%$. The stability of tolsultazolamide was assessed during all the storage steps of the experiment, including (a) in the plasma samples at $-20^{\circ} \mathrm{C}$ for 1 week, (b) in the plasma samples after two thaw cycles, and (c) in the extracted samples for $24 \mathrm{~h}$ at room temperature. It indicate that tolsultazolamide was stable under all the conditions as described above.

\section{Pharmacokinetic study}

\section{Pharmacokinetic study after intravenous administration}

The plasma concentration-time curves and the pharmacokinetic parameters of tolsultazolamide after its intravenous administration ( 1 or $2 \mathrm{mg} / \mathrm{kg}$ ) are presented in Figure 3A and Table 1, respectively. After its intravenous administration, tolsultazolamide was rapidly eliminated from the plasma, with $T_{1 / 2}$ of about $60-90 \mathrm{~min}$. The $\mathrm{AUC}_{0-\mathrm{t}}$ and the initial concentration $\left(C_{0}\right)$ values were proportional to the intravenous doses, with no statistical differences of the dose-normalized $\mathrm{AUC}_{0-\mathrm{t}}$ $\left(\mathrm{AUC}_{0-\mathrm{t}} / \mathrm{D}\right)$ and $\mathrm{C}_{0}\left(\mathrm{C}_{0} / \mathrm{D}\right)$ between the two doses $(P>0.05)$. However, other pharmacokinetic parameters such as $T_{1 / 2}, \mathrm{CL}$, and $V_{\mathrm{d}}$ were independent of the intravenous doses.

Table 1. Pharmacokinetic parameters of tolsultazolamide in rats after intravenous administration at two different doses. $n=6$. Mean \pm SD. ${ }^{b} P<0.05,{ }^{c} P<0.01$, comparison between the two groups.

\begin{tabular}{lcc}
\hline Parameters & $1 \mathrm{mg} / \mathrm{kg}$ & $2 \mathrm{mg} / \mathrm{kg}$ \\
\hline$k_{\mathrm{e}}(1 / \mathrm{min})$ & $0.010 \pm 0.006$ & $0.014 \pm 0.004$ \\
$T_{1 / 2}(\mathrm{~min})$ & $85.2 \pm 33.4$ & $54.0 \pm 20.2$ \\
$C_{0}(\mu \mathrm{g} / \mathrm{mL})$ & $1.922 \pm 0.293$ & $3.340 \pm 1.430^{\mathrm{b}}$ \\
$\mathrm{AUC} \mathrm{C}_{0-\mathrm{t}}(\mathrm{min} \cdot \mu \mathrm{g} / \mathrm{mL})$ & $14.169 \pm 2.543$ & $27.299 \pm 8.016^{c}$ \\
$\mathrm{AUC} \mathrm{C}_{-\infty}(\mathrm{min} \cdot \mu \mathrm{gg} / \mathrm{mL})$ & $17.992 \pm 3.835$ & $31.013 \pm 8.120^{\circ}$ \\
$V_{\mathrm{d}}(\mathrm{L} / \mathrm{kg})$ & $6.716 \pm 2.208$ & $5.400 \pm 2.556$ \\
$\mathrm{CL}\left(\mathrm{mL} \cdot \mathrm{min}^{-1} \cdot \mathrm{kg}^{-1}\right)$ & $57.803 \pm 12.708$ & $67.876 \pm 16.051$ \\
\hline
\end{tabular}

\section{Pharmacokinetic study after oral administration}

As shown in Table 2 and Figure 3B, after oral administration of tolsultazolamide at doses from 20 to $80 \mathrm{mg} / \mathrm{kg}, T_{\max }$, and

Table 2. Pharmacokinetic parameters of tolsultazolamide in rats after oral administration at three different doses. $n=6$. Mean \pm SD. ${ }^{c} P<0.01$ among the three groups.

\begin{tabular}{lccc}
\hline \multicolumn{1}{c}{ Parameters } & $20 \mathrm{mg} / \mathrm{kg}$ & $40 \mathrm{mg} / \mathrm{kg}$ & $80 \mathrm{mg} / \mathrm{kg}$ \\
\hline$k_{\mathrm{e}}(1 / \mathrm{h})$ & $0.122 \pm 0.059$ & $0.115 \pm 0.036$ & $0.154 \pm 0.046$ \\
$T_{1 / 2}(\mathrm{~h})$ & $6.8 \pm 3.1$ & $6.6 \pm 2.4$ & $4.8 \pm 1.3$ \\
$T_{\max }(\mathrm{h})$ & $2.2 \pm 0.5$ & $2.1 \pm 0.6$ & $1.8 \pm 0.5$ \\
$C_{\max }(\mu \mathrm{g} / \mathrm{mL})$ & $0.523 \pm 0.213$ & $0.710 \pm 0.245$ & $1.626 \pm 0.266^{\mathrm{c}}$ \\
$\mathrm{AUC} \mathrm{C}_{0-\mathrm{t}}(\mathrm{h} \cdot \mathrm{\mu g} / \mathrm{mL})$ & $2.730 \pm 1.290$ & $5.580 \pm 1.839$ & $11.438 \pm 1.637^{\circ}$ \\
$\mathrm{AUC} \mathrm{C}_{0-\infty}(\mathrm{h} \cdot \mu \mathrm{gg} / \mathrm{mL})$ & $3.893 \pm 2.024$ & $6.004 \pm 1.796$ & $12.278 \pm 2.533^{\mathrm{c}}$ \\
$V_{\mathrm{d} / \mathrm{F}(\mathrm{L} / \mathrm{kg})}$ & $60.019 \pm 37.779$ & $71.526 \pm 45.825$ & $45.925 \pm 12.909$ \\
$\mathrm{CL} / \mathrm{F}\left(\mathrm{L} \cdot \mathrm{h}^{-1} \cdot \mathrm{kg}^{-1}\right)$ & $6.444 \pm 3.352$ & $7.174 \pm 2.106$ & $6.740 \pm 1.324$ \\
$\mathrm{MRT}(\mathrm{h})$ & $4.8 \pm 0.3$ & $8.4 \pm 0.9$ & $7.5 \pm 1.3$ \\
\hline
\end{tabular}


A

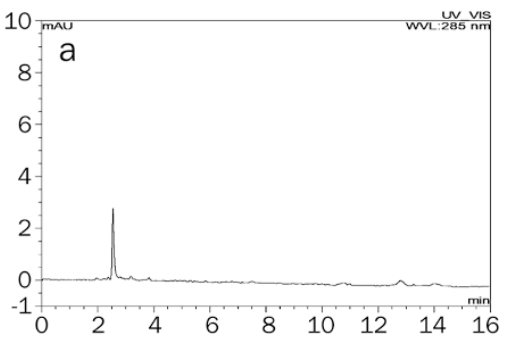

B

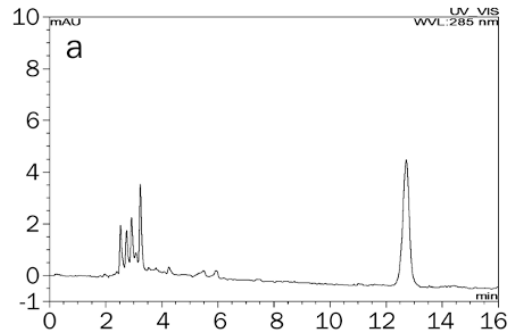

C

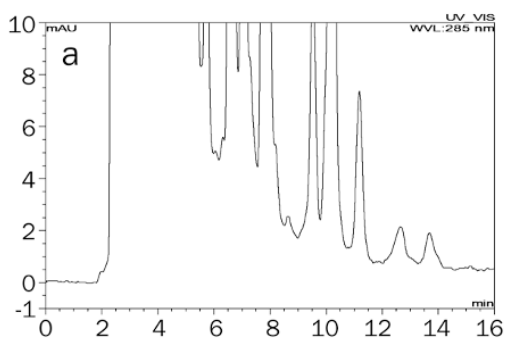

D

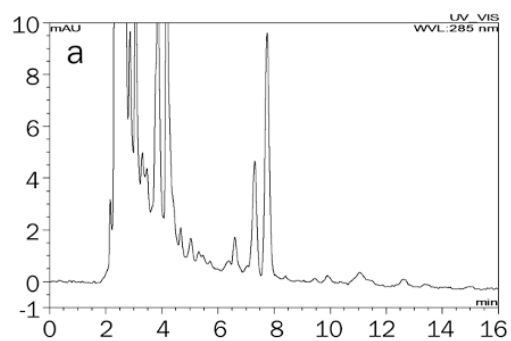

E

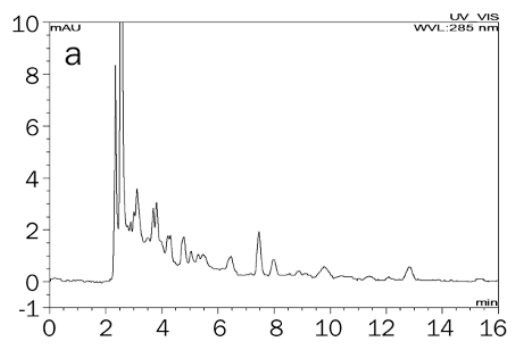

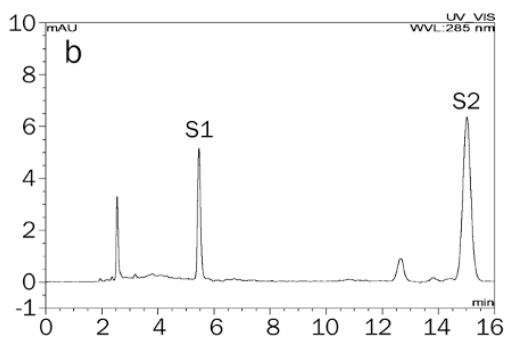
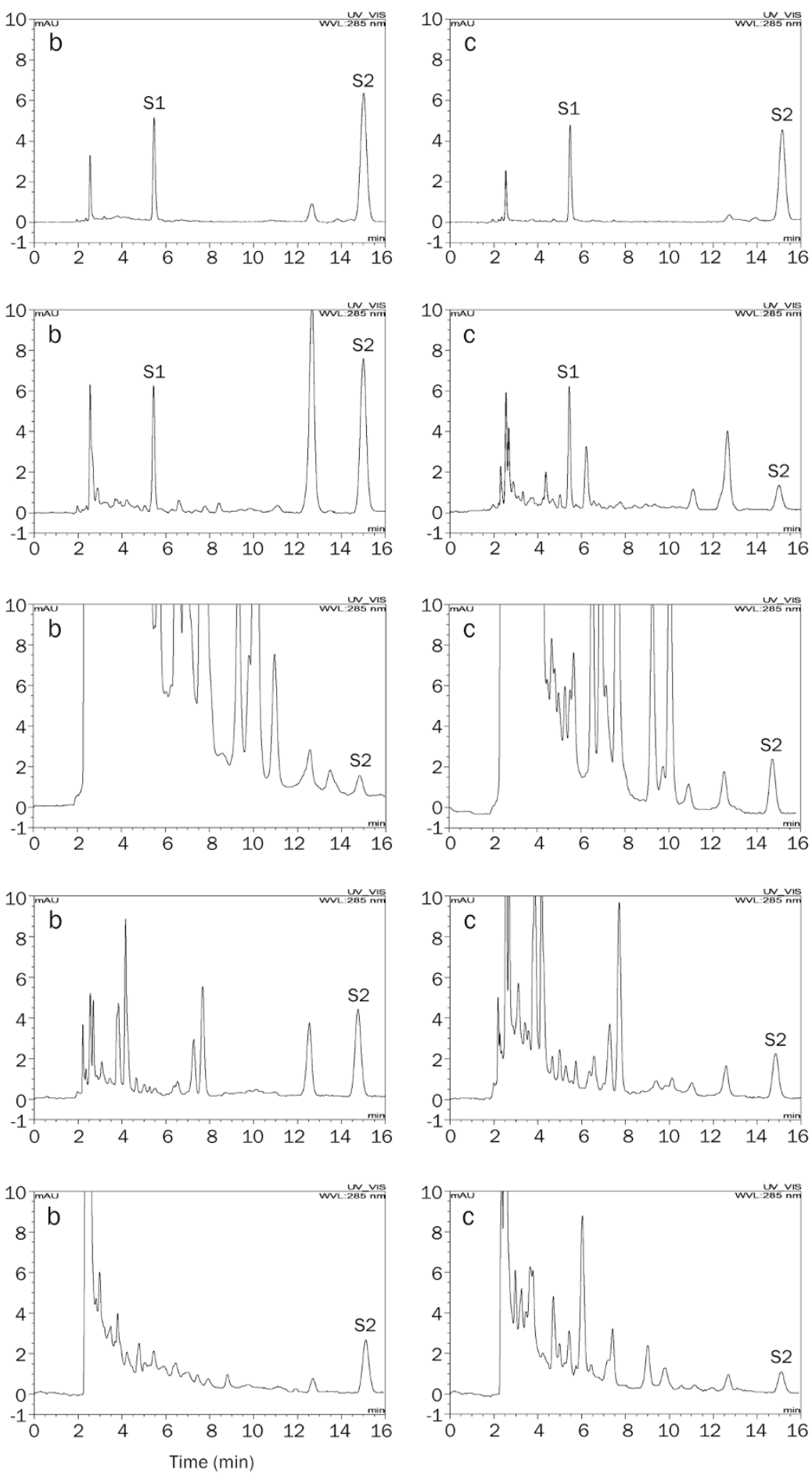

Figure 2. The HPLC chromatograms of tolsultazolamide in rat biological samples. (A-a) blank plasma; (A-b) blank plasma spiked with tolsultazolamide and IS; (A-C) actual plasma sample; (B-a) blank liver homogenate; (B-b) blank liver homogenate spiked with tolsultazolamide and IS; (B-C) actual liver homogenate sample; (C-a) blank urine; (C-b) blank urine spiked with tolsultazolamide; (C-C) actual urine sample; (D-a) blank feces homogenate; (D-b) blank feces homogenate spiked with tolsultazolamide; (D-c) actual feces homogenate sample; (E-a) blank bile; (E-b) blank bile spiked with tolsultazolamide; (E-c) actual bile sample. (S1, IS; S2, tolsultazolamide).

$T_{1 / 2}$ were both dose independent and were approximately $2 \mathrm{~h}$ and $5-7 \mathrm{~h}$, respectively. $\mathrm{AUC}_{0-\mathrm{t}}$ and dose, as well as $C_{\max }$ and dose, showed good linear relationships, with correlation coefficients $\left(r^{2}\right)$ of 1 and 0.985 , respectively. In addition, the dose-normalized $\mathrm{AUC}_{0-\mathrm{t}}\left(\mathrm{AUC}_{0-\mathrm{t}} / \mathrm{D}\right)$ and $C_{\max }\left(C_{\max } / \mathrm{D}\right)$ were not significantly different among the three doses analyzed by 

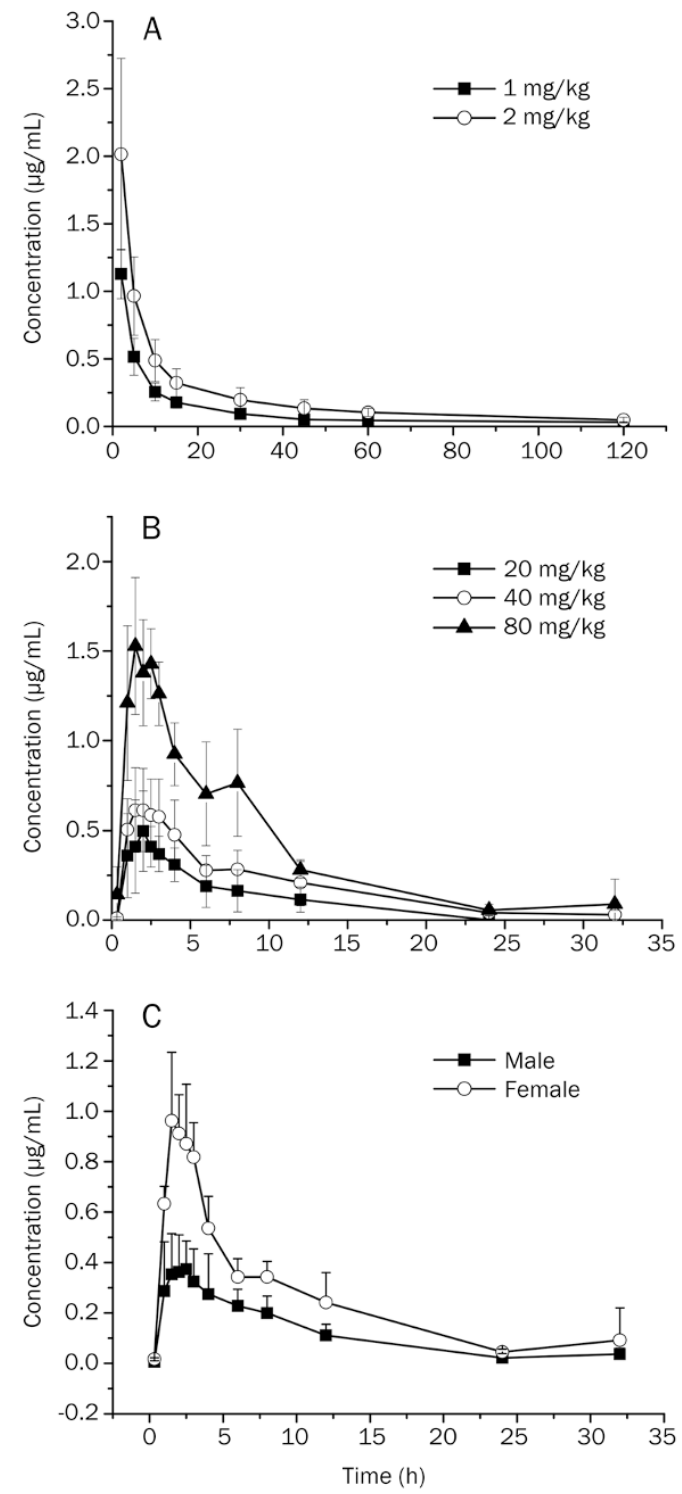

Figure 3. Plasma concentration-time profiles of tolsultazolamide in rats (mean $\pm S D$ ). (A) Pharmacokinetic profiles of 1 and $2 \mathrm{mg} / \mathrm{kg}$ tolsultazolamide administered intravenously $(n=6)$; (B) Pharmacokinetic profiles of 20,40 , and $80 \mathrm{mg} / \mathrm{kg}$ tolsultazolamide administered orally $(n=6)$; (C) Pharmacokinetic profiles of male and female rats after the oral administration of $40 \mathrm{mg} / \mathrm{kg}$ tolsultazolamide ( $n=5$ for each sex).

\section{ANOVA $(P>0.05)$.}

\section{Oral absolute bioavailability of tolsultazolamide}

The absolute bioavailability $F(\%)$ of tolsultazolamide was estimated with the following equation ${ }^{[14]}: F(\%)=\left[\left(\mathrm{AUC}_{\mathrm{oral}} \times\right.\right.$ Dose $\left._{\text {iv }}\right) /\left(\mathrm{AUC}_{\mathrm{iv}} \times\right.$ Dose $\left.\left._{\text {oral }}\right)\right] \times 100 \%$, where $\mathrm{AUC}_{\text {oral }}$ and $\mathrm{AUC}_{\mathrm{iv}}$ are the areas under the concentration-time curves and Dose $\mathrm{iv}_{\mathrm{iv}}$

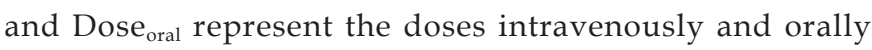
administered, respectively. The mean oral absolute bioavailability of tolsultazolamide administered at 20,40, and 80 $\mathrm{mg} / \mathrm{kg}$ was $58.91 \% \pm 1.56 \%, 60.02 \% \pm 1.59 \%$, and $61.70 \% \pm 1.63 \%$, respectively, and bioavailability was thus dose independent
$(P>0.05)$. The final average oral absolute bioavailability was $60.27 \% \pm 0.04 \%$.

\section{Sex differences in the pharmacokinetics of orally administered} tolsultazolamide

During our study of the oral pharmacokinetic properties of tolsultazolamide, we observed that the plasma concentrations of tolsultazolamide differed greatly between the male and female rats. Therefore, a further experiment was performed to investigate the influence of sex on the pharmacokinetics of tolsultazolamide. The pharmacokinetic parameters of orally administered tolsultazolamide $(40 \mathrm{mg} / \mathrm{kg}$ ) in each sex are given in Table 3, and the plasma concentration-time curves are shown in Figure 3C. Significant sex differences were observed in $C_{\max }$ and AUC, which were about twofold higher in the female rats than those in the male rats. In contrast, the $V_{\mathrm{d}} / \mathrm{F}$ and $\mathrm{CL} / \mathrm{F}$ values in the female rats were significantly lower than those in the male rats. In addition, the oral absolute bioavailability was also significantly different between female rats (about 83\%) and male rats (about 37\%). However, there was no significant difference between the sexes in $T_{\max }$ or $T_{1 / 2}$.

Table 3. Pharmacokinetic parameters of orally administered tolsultazolamide in different sexes. $n=5$. Mean \pm SD. ${ }^{b} P<0.05,{ }^{c} P<0.01$, comparison between the two groups.

\begin{tabular}{lcc}
\hline Parameter & Male & Female \\
\hline$k_{\mathrm{e}}(1 / \mathrm{h})$ & $0.097 \pm 0.050$ & $0.119 \pm 0.021$ \\
$T_{1 / 2}(\mathrm{~h})$ & $8.6 \pm 3.8$ & $6.0 \pm 1.1$ \\
$T_{\max }(\mathrm{h})$ & $2.9 \pm 1.8$ & $1.8 \pm 0.4$ \\
$C_{\max }(\mu \mathrm{g} / \mathrm{mL})$ & $0.421 \pm 0.130$ & $1.045 \pm 0.178^{\mathrm{c}}$ \\
$\mathrm{AUC} \mathrm{C}_{0-\mathrm{t}}(\mathrm{h} \cdot \mathrm{\mu g} / \mathrm{mL})$ & $3.452 \pm 1.010$ & $7.123 \pm 0.962^{\mathrm{c}}$ \\
$\mathrm{AUC} \mathrm{C}_{0-\infty}(\mathrm{h} \cdot \mu \mathrm{g} / \mathrm{mL})$ & $4.285 \pm 0.752$ & $8.062 \pm 1.373^{\mathrm{c}}$ \\
$V_{\mathrm{d}} / \mathrm{F}(\mathrm{L} / \mathrm{kg})$ & $122.762 \pm 61.235$ & $44.410 \pm 13.932^{\mathrm{b}}$ \\
$\mathrm{CL} / \mathrm{F}\left(\mathrm{L} \cdot \mathrm{h}^{-1} \cdot \mathrm{kg}^{-1}\right)$ & $9.606 \pm 1.938$ & $5.074 \pm 0.832^{\mathrm{c}}$ \\
$\mathrm{MRT}(\mathrm{h})$ & $8.6 \pm 2.0$ & $8.3 \pm 1.0$ \\
\hline
\end{tabular}

F: 37\% for male, $~ 83 \%$ for female.

\section{Tissue distribution}

The tissue distribution of tolsultazolamide was investigated following a single oral dose of $80 \mathrm{mg} / \mathrm{kg}$. The concentrations of tolsultazolamide in the various tissues were measured at $0.5,2,8$, and $24 \mathrm{~h}$ after its administration (Figure 4). Tolsultazolamide was identified in all the tissues tested $0.5 \mathrm{~h}$ after its administration, suggesting its rapid and extensive distribution. In most tissues, the concentration of tolsultazolamide reached a peak at $2 \mathrm{~h}$ and was still elevated $8 \mathrm{~h}$ after drug administration. The highest tolsultazolamide levels were detected in the stomach, intestine, spleen, lung, and kidney. By $24 \mathrm{~h}$, less than $50 \%$ of the maximum tolsultazolamide remained in most tissues.

\section{Excretion}

The excretion-time profile data for tolsultazolamide in the 


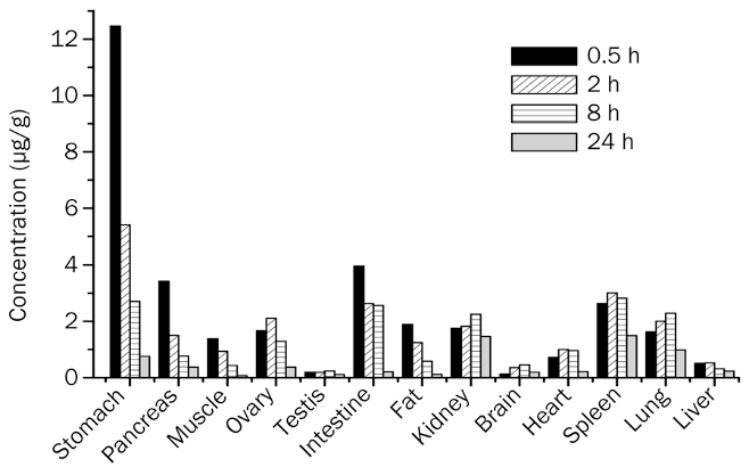

Figure 4. Tissue distribution of tolsultazolamide in rats after the oral administration of $80 \mathrm{mg} / \mathrm{kg}$ (mean \pm SD. $n=6$ ).

urine, feces, and bile after a single oral dose of $40 \mathrm{mg} / \mathrm{kg}$ are shown in Table 4 . The average cumulative excretion of tolsultazolamide was $0.016 \% \pm 0.019 \%$ in the urine and $1.115 \% \pm 0.506 \%$ in the feces within $72 \mathrm{~h}$, and $0.015 \% \pm 0.007 \%$ in the bile within $24 \mathrm{~h}$.

Table 4. Excretion of tolsultazolamide from urine, feces and bile in rats after oral administration. $n=6$. Mean \pm SD.

\begin{tabular}{lccc}
\hline Parameter & Urine (\%) & Feces (\%) & Bile (\%) \\
\hline $0-24 \mathrm{~h}$ & $0.007 \pm 0.010$ & $1.032 \pm 0.556$ & $0.015 \pm 0.007$ \\
$24-48 \mathrm{~h}$ & $0.006 \pm 0.005$ & $0.078 \pm 0.127$ & - \\
$48-72 \mathrm{~h}$ & $0.003 \pm 0.004$ & $0.005 \pm 0.006$ & - \\
Total & $0.016 \pm 0.019$ & $1.115 \pm 0.506$ & $0.015 \pm 0.007$ \\
\hline
\end{tabular}

\section{Discussion}

In this study, we first evaluated the pharmacokinetics, tissue distribution, and excretion properties of a novel carbonic anhydrase inhibitor, tolsultazolamide, in the rat. The results of this study should provide a meaningful basis for the use of tolsultazolamide as a new drug for the clinical prevention and treatment of AMS.

A rapid and sensitive high-performance liquid chromatography method, with simple liquid-liquid extraction, was established and validated for the quantitative analysis of tolsultazolamide in various biological samples. After its oral administration, both $\mathrm{AUC}_{0-\mathrm{t}}$ and $C_{\max }$ for tolsultazolamide showed perfect linear relationships with the oral dose, and there were no significant differences of the dose-normalized $\mathrm{AUC}_{0-\mathrm{t}}$ $\left(\mathrm{AUC}_{0-\mathrm{t}} / \mathrm{D}\right)$ and $C_{\max }\left(C_{\max } / \mathrm{D}\right)$ among the three doses $(20,40$, and $80 \mathrm{mg} / \mathrm{kg}$ ), whereas other pharmacokinetics parameters such as $T_{1 / 2}, \mathrm{CL}$, and $V_{\mathrm{d}}$ were dose independent. These findings support linear rather than non-linear pharmacokinetic profiles of tolsultazolamide in rats within the dose range studied. The value of $\mathrm{CL} / \mathrm{F}$ was larger than $100 \mathrm{~mL} \cdot \mathrm{min}^{-1} \cdot \mathrm{kg}^{-1}$, which was about twofold of the hepatic blood flow rate (about $\left.55.2 \mathrm{~mL} \cdot \mathrm{min}^{-1} \cdot \mathrm{kg}^{-1}\right)^{[15]}$, suggesting a rapid clearance of tolsult- azolamide from the rat body. The value of $V_{\mathrm{d}} / \mathrm{F}$ was higher than $45000 \mathrm{~mL} / \mathrm{kg}$, which was much larger than the total blood volume (about $54.0 \mathrm{~mL} / \mathrm{kg}$ ) and the total body water in rat (about $671.0 \mathrm{~mL} / \mathrm{kg})^{[15]}$, demonstrating a wide distribution of tolsultazolamide into the extravascular systems. The average oral absolute bioavailability of tolsultazolamide in the rat was about $60 \%$, which demonstrated that tolsultazolamide is absorbed well in rats.

The tissue distribution of a drug is vital when investigating its major target sites and interpreting its disposition in vivo ${ }^{[16]}$. In our tissue distribution study, tolsultazolamide presented an extensive distribution profile and was detected in all the tissues examined, which were consistent with the results of $V_{\mathrm{d}} / \mathrm{F}$ in the pharmacokinetic study. After its oral administration, the concentrations of tolsultazolamide peaked at $2 \mathrm{~h}$ in most tissues and declined dramatically by $24 \mathrm{~h}$, indicating that there was no apparent accumulation of tolsultazolamide in tissues. The $\mathrm{AUC}_{0-24}$ in tissues was in descending order of stomach, spleen, kidney, intestine, lung, ovary, pancreas, plasma, heart, fat, muscle, brain, liver and testis (data not shown). The $\mathrm{AUC}_{0-24}$ of brain was about $44 \%$ of that in plasma, suggesting that the lipid solubility enabled tolsultazolamide to cross the blood-brain barrier. Furthermore, the relatively high distributions of tolsultazolamide in kidney and lung may contribute to its use for the prevention and treatment of AMS, for the kidney is regarded as the major target organ of acetazolamide in the prophylaxis and treatment of AMS and the lung is the major pathological organ in $\mathrm{AMS}^{[17,18]}$.

In our excretion experiments, we estimated the unchanged fraction of tolsultazolamide in the urine, feces, and bile. Only a small proportion (less than $2 \%$ ) of tolsultazolamide was excreted intact, demonstrating the extensive metabolism of this drug after its oral administration in rats. Further studies are required to investigate the pathways of tolsultazolamide metabolism and to clarify whether tolsultazolamide itself or its metabolites make the major contribution to its significant pharmacological effects.

In recent years, the importance of sex differences in drug studies has been recognized ${ }^{[19-21]}$, and consideration of these differences increases the safety and efficacy of drug-based therapies. In the present study, we found significant sex-based differences in the pharmacokinetics of orally administered tolsultazolamide, in that the concentrations of tolsultazolamide were almost higher in female rats than those in male rats at each time point. The $C_{\max }$ and AUC values in female rats were both about twofold higher than those in male rats, whereas the $V_{\mathrm{d}} / \mathrm{F}$ and CL/F values in female rats were significantly lower than those in male rats $(P<0.05)$. However, we did not observe significant differences in AUC values between female and male rats after intravenous administration of tolsultazolamide, so the oral absolute bioavailability of female rats was higher than that of male rats (about $83 \%$ and $37 \%$, respectively), which would also cause the sex differences in CL/F and $V_{\mathrm{d}} / \mathrm{F}$ after oral administration of tolsultazolamide. These results suggest that the observed sex differences in response to orally administered tolsultazolamide were probably caused 
by the difference in oral absolute bioavailability between female and male rats. In addition, many other mechanisms could also underlie the sex-based differences, including physiological factors such as body weight, organ size, glomerular filtration, and bowel motility, and molecular factors, such as specific transporters and drug-metabolizing enzymes ${ }^{[20,21]}$. In this study, the concentrations of tolsultazolamide in the kidneys also differed between the sexes, and were almost twofold higher in the female rats than those in the male rats at all the time points examined (data not shown). Therefore, further studies are required to explore the exact reasons for the sexbased differences in the pharmacokinetics of tolsultazolamide in rats and to investigate whether sex significantly influences the pharmacokinetics of tolsultazolamide in humans.

Tolsultazolamide is a derivative of acetazolamide, so it is very meaningful to compare its pharmacokinetics, tissue distribution, and excretion characteristics with acetazolamide. The $T_{\max }$ and $T_{1 / 2}$ of tolsultazolamide (about $2 \mathrm{~h}$ and 5-7 h, respectively) were very close to those of acetazolamide (about 1-3 $\mathrm{h}$ and $6 \mathrm{~h}$, respectively). Moreover, both of them were well absorbed following oral administration, and widely distributed to body tissues. However, only a small proportion (less than $2 \%$ ) of tolsultazolamide was excreted intact, which is very different from acetazolamide (most excreted unchanged in urine) $)^{[22]}$.

Taken together, the achieved pharmacokinetics, tissue distribution and excretion results of tolsultazolamide may be useful for better understanding of the pharmacodynamics of tolsultazolamide and its mechanism of action, such as when and where it can be effective, and these results will also provide important information for evaluation of the safety of tolsultazolamide. Furthermore, these data will provide theoretical basis for designing drug treatment regiments of the clinical trials.

However, there were also some limitations of our study. In the current study, we did not study the effects of acute exposure to high altitude on the pharmacokinetics of tolsultazolamide. It is known that immediate exposure to high altitude will induce some physiologic changes, such as the redistribution of blood flow to the most metabolically active systems and the changes of the content of protein and red blood cell, and these physiologic changes may alter drug pharmacokinetics that, in turn, might require modifications in dosage regimens to maintain efficacy or prevent toxicity. Previous study has reported an increased clearance of acetazolamide after acute and chronic exposure to $4360 \mathrm{~m}^{[23]}$. Therefore, further studies are required to investigate the high-altitude pharmacokinetics characteristics of tolsultazolamide. In addition, the major metabolic products and pathways of tolsultazolamide were not investigated in the present study, which also need to be explored in the future studies.

\section{Conclusions}

This is the first study to evaluate the pharmacokinetics, tissue distribution, and excretion characteristics of tolsultazolamide in rats. Following its oral administration, tolsultazolamide was rapidly absorbed and showed relatively high bioavailability. The tissue distribution of tolsultazolamide was established rapidly and was widespread, but the total recovery of unchanged tolsultazolamide in the urine, feces, and bile was very low, indicating the extensive metabolism of tolsultazolamide. Sex also significantly affected the pharmacokinetics of orally administered tolsultazolamide. These results provide reliable scientific data, which helps to design safe and effective treatment regimens for the clinical administration of the drug.

\section{Supplementary Information}

Supplementary information was available on the Acta Pharmacologica Sinica's website.

\section{Acknowledgements}

This work was supported by grant from the State Key Project of Drug Research and Development of China (Grant No 2012ZX09J12109-01B).

\section{Author contribution}

Jin-da WANG, Yong-ping SHI, Wen-yu CUI, Yan-fang ZHANG, and Hai WANG designed the research; Jin-da WANG, Jing YIN, Zhi-yuan PAN, and Yong-ping SHI performed the research; Jing YIN and Yan-fang ZHANG contributed new reagents; Jin-da WANG, Wen-yu CUI, and Zhi-yuan PAN analyzed data; Jin-da WANG, Yong-ping SHI, and Hai WANG wrote the paper.

\section{References}

1 Roach RC, Hackett PH. Frontiers of hypoxia research: acute mountain sickness. J Exp Biol 2001; 204: 3161-70.

2 Clarke C. Acute mountain sickness: medical problems associated with acute and subacute exposure to hypobaric hypoxia. Postgrad Med J 2006; 82: 748-53.

3 Imray C, Wright A, Subudhi A, Roach R. Acute mountain sickness: pathophysiology, prevention, and treatment. Prog Cardiovasc Dis 2010; 52: 467-84.

4 Murdoch D. Altitude sickness. Clin Evid 2010; 3: 1209-19.

5 Kayser B, Dumont L, Lysakowski C, Combescure C, Haller G, Tramèr MR. Reappraisal of acetazolamide for the prevention of acute mountain sickness: a systematic review and meta-analysis. High Alt Med Biol 2012; 13: 82-92.

6 Low EV, Avery AJ, Gupta V. Identifying the lowest effective dose of acetazolamide for the prophylaxis of acute mountain sickness: systematic review and meta-analysis. BMJ 2012; 345: e6779.

7 Ritchie ND, Baggott AV, Andrew Todd WT. Acetazolamide for the prevention of acute mountain sickness - A systematic review and meta-analysis. J Travel Med 2012; 19: 298-307.

8 Seupaul RA, Welch JL, Malka ST, Emmett TW. Pharmacologic prophylaxis for acute mountain sickness: a systematic shortcut review. Ann Emerg Med 2012; 59: 307-17.

9 Shu YG, Zhang DX, Xiao ZH, Cui WY, Nie HJ, Zhang YK, et al. Effect of neotype carbonic anhydrase target-based inhibitors (P-8) on the hypoxic tolerance in mice. Chin J Appl Physiol 2011; 27: 276-9.

10 Xiao ZH, Duan RF, Cui WY, Zhang YF, Zhang SG, Chen FJ, et al. Synthesis and evaluation of new carbonic anhydrase inhibitors. Bioorg Med Chem 2011; 19: 3221-8.

11 He CY, Wang S, Feng Y, Liang S, Lin X, Xu DS, et al. Pharmacokinetic, 
tissue distribution and metabolism of senkyunolide I, a major bioactive component in Ligusticum chuanxiong Hort (Umbelliferae). J Ethnopharmacol 2012; 142: 706-13.

12 Zhang Z, Liu YY, Su MQ, Liang XF, Wang WF, Zhu X. Pharmacokinetics, tissue distribution and excretion study of $d /$-praeruptorin $A$ of Peucedanum praeruptorum in rats by liquid chromatography tandem mass spectrometry. Phytomedicine 2011; 18: 527-32.

13 Lee SH, Gu N, Kim BH, Lim KS, Shin SG, Jang IJ, et al. Pharmacokinetic and pharmacodynamics properties of the calcimimetic agent cinacalcet (KRN1493) in healthy male Korean subjects: a randomized, open-label, single ascending-dose, parallel-group study. Clin Ther 2012; 34: 1160-9.

14 Geng T, Sun Y, Yao WF, Ding AW, Zhang L, Guo JM, et al. Pharmacokinetics and tissue distribution of schizonepetin in rats. Fitoterapia 2011; 82: 1110-7.

15 LV GY, Lou ZH, Chen SH, Gu H, Shan LT. Pharmacokinetics and tissue distribution of 2,3,5,4'-tetrahydroxystilbene-2-0- $\beta$ - $D$-glucoside from traditional Chinese medicine Polygonum multiflorum following oral administration to rats. J Ethnopharmacol 2011; 137: 449-56.

16 Yin QW, Sun H, Zhang AH, Wang XJ. Pharmacokinetics and tissue distribution study of scoparone in rats by ultraperformance liquid- chromatography with tandem high-definition mass spectrometry. Fitoterapia 2012; 83: 795-800.

17 Leaf DE, Goldfarb DS. Mechanisms of action of acetazolamide in the prophylaxis and treatment of acute mountain sickness. J Appl Physiol 2007; 102: 1313-22.

18 Swenson ER. Prevention of acute mountain sickness by acetazolamide: as yet an unfinished story. J Appl Physiol 2007; 102: 1305-7.

19 Franconi F, Brunelleschi S, Steardo L, Cuomo V. Gender differences in drug responses. Pharmacol Res 2007; 55: 81-95.

20 Schwartz JB. The influence of sex on pharmacokinetics. Clin Pharmacokinet 2003; 42: 107-21.

21 Soldin OP, Mattison DR. Sex differences in pharmacokinetics and pharmacodynamics. Clin Pharmacokinet 2009; 48: 143-57.

22 Rankin GO. Acetazolamide. In: Enna SJ, Bylund DB, Editors-in-Chief. Xpharm: the comprehensive pharmacology reference. Elsevier Inc. 2007. p 1-5.

23 Li XY, Gao F, Li ZQ, Guan W, Feng WL, Ge RL. Comparison of the pharmacokinetics of sulfamethoxazole in male Chinese volunteers at low altitude and acute exposure to high altitude versus subjects living chronically at high altitude: an open-label, controlled, prospective study. Clin Ther 2009; 31: 2744-54. 